\section{Possible technical and biological explanations for the "parental telomere length inheritance discrepancy' enigma}

European Journal of Human Genetics (2015) 23, 3-4; doi:10.1038/ejhg.2014.65; published online 23 April 2014

Telomeres, the repetitive nucleoprotein complexes capping the ends of human chromosomes, are highly heritable but also diminish in length with aging and due to environmental factors such as smoking. ${ }^{1}$ Critically short telomeres signal replicative senescence, and shorter telomeres have been reported as a risk factor for cancer, cardiovascular and infectious disease, ${ }^{2-5}$ although causality remains to be validated. Whereas telomere length (TL) has a high heritability, the inheritance patterns of TL exhibit peculiarities that require further examination. Initial smaller studies suggested that paternal inheritance was the primary determining factor of offspring's TL. ${ }^{6-8}$ However, the recent large-scale study by Broer et al ${ }^{9}$ in this journal demonstrated both maternal and paternal inheritance, but with a significantly stronger maternal component.

This important report inspired discussion in the field, leading to two independent commentaries by the authors of this Letter. In the first commentary, Eisenberg ${ }^{10}$ demonstrated high levels of heterogeneity between the different published studies in the field that is, in some populations there is stronger maternal TL inheritance, whereas in others paternal inheritance is more important. Here, we refer to this as the 'parental TL inheritance discrepancy'. In a second commentary, De Meyer et al ${ }^{11}$ discussed the consequences of the study: offspring TL might be predominantly determined by the TL of the sperm and egg cells at the offspring's conception. Although additional genetic modulation is present, particularly in the telomerase machinery re-elongating telomeres in germ cells and zygote, its impact appears to be limited. ${ }^{12}$ As the parental TL inheritance discrepancy was central to both commentaries, an editorial initiative launched a discussion between the authors. Inspired by this discussion, here, different mechanisms potentially explaining the parental TL inheritance discrepancy are presented. We propose that stronger maternal or paternal inheritance in a specific cohort will be the net effect of these and additional unknown mechanisms depending on the specific design of the study.

In explaining the discrepancy, putative biological effects (except imprinting) may be particularly present during gametogenesis, whereas putative methodological effects will typically arise after birth. Keeping this order in mind, potential biological effects are presented first. In the case of direct transmission of TL to the offspring through the germ cell TLs, random variability of the germ cell TL might dilute the correlations between blood TL measured in parents and offspring. ${ }^{11}$ Sperm cell progenitors keep dividing throughout life, whereas more limited numbers of primary oocytes are available from birth. Stochastic effects, for example, associated with androgen fluctuations influencing testicular telomerase activity, ${ }^{13}$ might therefore lead to more random fluctuations in sperm cell TL than in oocyte TL. This greater variability in sperm cell TL would lessen the paternal blood-sperm cell TL correspondence and consequently lower the correlation between paternal and offspring blood TL. If these sources of variability cumulatively accrue with age, the fatheroffspring correlation would tend to progressively lessen for offspring conceived by older fathers, whereas maternal-offspring correlations should be less influenced by maternal age at conception.

Contrasting the correlation diluting influence of 'random' variability, factors shared between offspring and parents might increase parent-specific TL associations. For example, random environmental phenomena affecting telomere biology during pregnancy will be particularly shared between mothers (but not fathers) and offspring, potentially leading to a higher maternal-offspring TL correlation. In addition to environmental effects, also shared genetic effects might explain the parental TL inheritance discrepancy. Specifically, alleles influencing TL maintenance are likely to affect the TL of somatic tissues throughout life. Despite an overall limited effect, these same alleles may have a larger influence on the TL of gametes in males than females, since male gametes continue to be produced throughout life. Therefore, genetic effects on TL maintenance in males might tend to cause somatic (eg, leukocyte) and gametic/offspring TL to resemble each other more than in females, particularly in case of older parents. Finally, as suggested and discussed earlier, ${ }^{7}$ also imprinting mechanisms could be responsible for specific parental effects.

In addition to possible biological mechanisms explaining this discrepancy, methodological issues need also be considered. Paternal and maternal TL inheritance has been typically reported as correlation of 'current' parental TL and offspring TL. This implies that any variance arising between conception and time of measurement affects the correlation (see Nordfjall et $a l^{6}$ ), and may result in a biased comparison if these factors differ in fathers and mothers. For example, TLs may be more similar in subjects living together due to a shared lifestyle, ${ }^{9}$ and a bias could arise if offspring predominantly live together with their mother upon parental divorce. As well, maternal education is often more strongly correlated with child health than paternal education, suggesting that children's lifestyle may more closely parallel maternal lifestyle than paternal lifestyle. ${ }^{14}$ This effect would particularly increase with older offspring. Conversely, varying non-paternity rates between populations might impact inheritance estimates already at birth. However, the two studies that report ruling out non-paternity ${ }^{1,9}$ are also the only studies not supporting stronger paternal-offspring TL correlations, the opposite of what would be expected if non-paternity was causing the discrepancy.

An additional technical explanation for the parental inheritance discrepancy is the sometimes observed nonlinearities in qPCR measurement scales (used in all but one ${ }^{1}$ inheritance study). ${ }^{15}$ Even in complete family trios without any sampling biases fathers have on average shorter TLs than mothers. If qPCR artifactually scales a difference in TL as larger the longer the TL is, then the degree of the (linear) Pearson correlation will particularly decrease for fatheroffspring pairs as these have on average the largest difference in TL. The impact will depend on the TL itself and therefore the age of the population. As well the nonlinearity in qPCR measurements may vary across labs. ${ }^{15}$ Finally, also a survival bias might be present, as exemplified by the fact that more mother-offspring pairs than father-offspring pairs were included in all available paternal-offspring 
TL studies (paired $t$-test, $P=0.05$, Table 1 by Eisenberg ${ }^{10}$ ). If lower paternal survival is associated with increased incidence of critically short TLs, the paternal TL distribution will be truncated leading to lower father-offspring correlations in older populations. Tentatively suggestive that this explanation is worthy of further consideration, across the limited set of available populations $(n=8)$, there is a nonsignificant trend toward greater fatheroffspring correlations (relative to mother-offspring correlations, difference) in studies with increasing numbers of father-offspring pairs (relative to mother-offspring pairs, ratio; Pearson correlation, $r=0.37, P=0.37)$.

As suggested above, biological phenomena may provide particularly important explanations for the inheritance discrepancy before fertilization, whereas methodology-associated biases may arise more after birth. A large-scale TL inheritance study of neonatal offspring along with both parents might identify whether biology or methodology lies at the origin of the observed discrepancy. Methodology-induced biases will most likely be related to measurement method, parental age at conception, parental and offspring age at measurement, and parental survival. Statistical adjustment for the latter variables, or selection of appropriate population subsets might allow discernment of the effect(s) at play. The use of nonparametric statistics or bettervalidated TL measurements might eliminate the possibility that the discrepancies are related to nonlinearity in the TL measurement scale. In summary, there are several possible explanations for the parental TL inheritance discrepancy, and the net effect most likely depends on the specific study design and characteristics of the included populations. A novel (meta-)analysis of the different data sets adjusting for the above-suggested putative confounding variables might enable the identification of the underlying mechanism, or at least might eliminate several methodological possibilities.

\section{CONFLICT OF INTEREST}

The authors declare no conflict of interest.

Tim De Meyer ${ }^{*}, 1$ and Dan TA Eisenberg ${ }^{2,3}$

${ }^{1}$ Department of Mathematical Modelling, Statistics and Bioinformatics, Ghent University, Ghent, Belgium;
${ }^{2}$ Department of Anthropology, University of Washington, Seattle, WA, USA;

${ }^{3}$ Center for Studies in Demography and Ecology, University of Washington, Seattle, WA, USA

E-mail: Tim.DeMeyer@ugent.be

1 Nawrot TS, Staessen JA, Gardner JP, Aviv A: Telomere length and possible link to X chromosome. Lancet 2004; 363: 507-510.

2 Willeit P, Willeit J, Kloss-Brandstatter A, Kronenberg F, Kiechl S: Fifteen-year follow-up of association between telomere length and incident cancer and cancer mortality. JAMA 2011; 306: 42-44.

3 Cawthon RM, Smith KR, O'Brien E, Sivatchenko A, Kerber RA: Association between telomere length in blood and mortality in people aged 60 years or older. Lancet 2003; 361: 393-395.

4 Cohen S, Janicki-Deverts D, Turner RB et al: Association between telomere length and experimentally induced upper respiratory viral infection in healthy adults. JAMA 2013 309: 699-705.

5 Brouilette SW, Moore JS, McMahon AD et al: Telomere length, risk of coronary heart disease, and statin treatment in the West of Scotland Primary Prevention Study: a nested case-control study. Lancet 2007; 369: 107-114.

6 Nordfjall K, Svenson U, Norrback KF, Adolfsson R, Roos G: Large-scale parent-child comparison confirms a strong paternal influence on telomere length. Eur J Hum Genet 2010; 18: 385-389.

7 Nordfjall K, Larefalk A, Lindgren P, Holmberg D, Roos G: Telomere length and heredity: indications of paternal inheritance. Proc Natl Acad Sci USA 2005; 102 : 16374-16378.

8 Njajou OT, Cawthon RM, Damcott CM et al: Telomere length is paternally inherited and is associated with parental lifespan. Proc Natl Acad Sci USA 2007; 104: 12135-12139.

9 Broer L, Codd V, Nyholt DR et al: Meta-analysis of telomere length in 19713 subjects reveals high heritability, stronger maternal inheritance and a paternal age effect. Eur J Hum Genet 2013; 21: 1163-1168.

10 Eisenberg DT: Inconsistent inheritance of telomere length (TL): is offspring TL more strongly correlated with maternal or paternal TL? Eur J Hum Genet 2013; 22: 8-9.

11 De Meyer T, Vandepitte K, Denil S, De Buyzere ML, Rietzschel ER, Bekaert S: A non-genetic, epigenetic-like mechanism of telomere length inheritance? Eur J Hum Genet 2013; 22: 10-11.

12 Codd V, Mangino M, van der Harst P et al: Common variants near TERC are associated with mean telomere length. Nat Genet 2010; 42: 197-199.

13 Eisenberg DT: An evolutionary review of human telomere biology: the thrifty telomere hypothesis and notes on potential adaptive paternal effects. Am J Human Biol 2011; 23: 149-167.

14 Cochrane SH, Leslie J, O'Hara DJ: Parental education and child health: intracountry evidence. Health Policy Educ 1982; 2: 213-250.

15 Elbers CC, Garcia ME, Kimura M et al: Comparison between Southern blots and qPCR analysis of leukocyte telomere length in the Health ABC Study. J Gerontol A Biol SCi Med Sci 2013; e-pub ahead of print 14 August 2013.

currently in use can be strongly influenced by the results of parental studies. ${ }^{1,3,4}$ We used a simple Bayesian model to demonstrate that in the case of inherited variants, the observed parental phenotype will be heavily and predictably influenced by the decreased reproductive fitness associated with disease expression. This means that transmitting parents with high penetrance variants will nonetheless often be 'unaffected'.

\section{BIASES INHERENT IN PARENTAL STUDIES}

In any individual most genetic variants, including rare variants, are inherited. ${ }^{5}$ Pathogenicity of very rare or private inherited CNVs is often inferred in part from the parental phenotype. Disease concordance between parent and offspring may be used to conclude that a shared CNV likely had a causal role, despite the fact that the $a$ priori probability of both the parent and a child sharing that $\mathrm{CNV}$ (given that the parent had the CNV) was 50\% in most cases. Disease discordance in the form of an 'unaffected' or 'healthy' parent, on the other hand, may be used to argue that a shared variant is likely benign. However, by definition, transmitting parents have been able to find a partner and to reproduce (and were available and willing to many developmental and neuropsychiatric conditions. The growing uptake of chromosomal microarray analysis in clinical practice ${ }^{1,2}$ is predicated on the assumption that there exist valid protocols to guide the interpretation of very rare or private CNVs. Many protocols 